\title{
Der Einfluss weitreichender Netzwerke auf die Plastizität der Großhirnrinde
}

\author{
Franziska Greifzu, Fred Wolf und Siegrid Löwel
}

\begin{abstract}
Zusammenfassung
Neuronale Plastizität gibt unserem Gehirn die Möglichkeit, sich das ganze Leben lang an neue Anforderungen anzupassen. Plastizität im erwachsenen Gehirn ist jedoch oft eingeschränkt und das Lernen mühsamer als in der Jugend. Wir untersuchen mit einer Kombination von Verhaltenstests und einer minimal-invasiven Messung von Hirnaktivität am Modell des Sehsystems von Mäusen, wie sich Lernen und Plastizität mit dem Alter und nach Läsionen verändern und die Struktur und Funktion von Nervenzellnetzwerken modifizieren. Wir hoffen, so nicht nur die Mechanismen von Gehirnentwicklung, Funktion und Lernen besser zu verstehen, sondern auch neue klinisch relevante Ansätze zur Förderung von Regeneration und Rehabilitation nach Hirnschädigungen entwickeln zu können. Unsere bisherigen Untersuchungen haben klare Hinweise für den Einfluss weitreichender Netzwerke auf die Funktion und Plastizität der Hirnrinde erbracht: Sie spielen eine wichtige Rolle für die Entwicklung der funktionellen Architektur der Sehrinde, und Läsionen in einem Hirnareal verändern nicht nur die Funktion des direkt betroffenen Areals, sondern haben über diese Netzwerke auch Auswirkungen auf weiter entfernt liegende Areale, sogar auf der gegenüberliegenden Hirnhälfte.
\end{abstract}

\begin{abstract}
Network influences on cortical plasticity.

Neuronal plasticity is the basis of our lifelong ability to learn and adapt to new challenges. Plasticity in adulthood is - however - often limited and learning more laborious than earlier on. Using a combination of behavioral tests and imaging of brain activity, we investigate in the visual system of mice, how learning and plasticity change during ageing and after lesions and modify the structure and function of nerve cell networks. We hope that answering these key questions not only helps to understand the rules underlying brain development, functioning and learning but additionally will open up new avenues to develop clinically relevant concepts to promote regeneration and rehabilitation for diseased and injured brains. Our research has revealed clear evidence for a prominent influence of long-range neuronal interactions on cortical function and plasticity: they play a major role for the development of functional cortical architecture, and lesions in one cortical area not only affect the function of the directly lesioned area but also distant areas, even on the opposite brain hemisphere.
\end{abstract}

Keywords: visual cortex; ageing; ocular dominance; stroke; visual acuity

\section{Flexibles Gehirn}

In der Abteilung ,Systems Neuroscience“ und dem BMBF-geförderten Konsortium Bernstein Fokus Lernen erforschen wir die Mechanismen neuronaler Plastizität in der Hirnrinde von Säugetieren, und zwar insbesondere im erwachsenen Gehirn, also nach den Phasen besonders ausgeprägter Plastizität in der frühen Jugend, im alternden Gehirn und nach Erkrankungen oder Läsionen des Nervensystems. Wir untersuchen neuronale Plastizität vor allem am Modell des Sehsystems von Mäusen, da dies die Möglichkeit bietet, unterschiedlich gentechnisch veränderte Tiere analysieren zu können. Damit werden ganz neue Einblicke auch in zelluläre und molekulare Mechanismen neuronaler Plastizität möglich.

Das Sehsystem von Mäusen ist in vieler Hinsicht dem Sehsystem von Menschen ähnlich: Informationen über Sehreize werden von der mehrschichtigen Netzhaut (Retina) in Form von Aktionspotenzialmustern im Sehnerv zunächst zu einem Kerngebiet im Thalamus, dem seitlichen Kniehöcker (Corpus geniculatum laterale), und von dort weiter in die primäre Sehrinde (=V1, visueller Kortex) geleitet (Abbildung 1A). Hierbei kreuzen die Sehnerven des nasalen Netzhautteils im Chiasma opticum (Sehnervenkreuzung) auf die gegenüberliegende Hirnhälfte, während Fasern aus dem temporalen (äußeren) Teil der Netzhaut nicht kreuzen. Dadurch erreichen Informationen aus der rechten Gesichtsfeldhälfte beider Augen die linke Hirnhälfte (Hemisphäre) und umgekehrt. Auf dem gesamten Weg bleiben Nachbarschaftsbeziehungen erhalten, das heißt, benachbarte Reize im Gesichtsfeld aktivieren auch benachbarte Zellen in V1. Diese Art der geordneten Abbildung einer peripheren Rezeptoroberfläche nennt man Retinotopie.

\section{Augendominanzplastizität und ihre Messung}

Der primäre visuelle Kortex teilt sich in einen monokularen (von griechisch monos "ein“" und lateinisch oculus „Auge“) und einen binokularen (,beidäugigen“) Teil. Monokulare Nervenzellen werden nur durch Stimulierung eines Auges aktiviert, binokulare durch beide Augen. Die klassischen Untersuchungen von Wiesel und Hubel haben gezeigt, dass frühe Erfahrung einen massiven Einfluss auf Struktur und Funktion der Nervennetzwerke in der Sehrinde hat (Wiesel und Hubel 1963). Die Unterdrückung der normalen beidäugigen Seherfahrung durch den Verschluss eines Auges (monokulare Deprivation $=\mathrm{MD}$, ein experimentelles Modell des grauen Stars) während einer frühen Phase der postnatalen Entwicklung führt in ihren Experimenten zu einer irreversiblen Änderung im Netzwerk der Nervenzellen der Sehrinde: Neurone, die normalerweise von beiden Augen aktiviert werden, reagierten nur noch auf Reizung des offenen Auges; ihre sogenannte „Augendominanz" (auch okuläre Dominanz, englisch ocular dominance, $\mathrm{OD}$ ) hatte sich verschoben, was man als Augendominanzplastizität bezeichnet. Diese Augendominanzplastizität ist das meist untersuchte Modell für Plastizität in der Hirnrinde. Im Unterschied zum Menschen wird V1 bei der Maus durch Eingänge des jeweils gegenüberliegenden (kontralateralen) Auges dominiert und das binokulare, von beiden Augen gesehene Gesichtsfeld ist nur etwa $30^{\circ}$ (Mensch: circa $120^{\circ}$ ) groß (Dräger 1975). Verschließt man bei einer Maus das „stärkere“ kontralaterale Auge, verschiebt sich auch hier die Augendominanz (Dräger 1978), und V1 wird anschließend in etwa gleich stark durch Reizung beider Augen aktiviert (Gordon und Stryker 1996). Auch Mäuse zeigen damit Augendominanzplastizität; dies ist genau die 


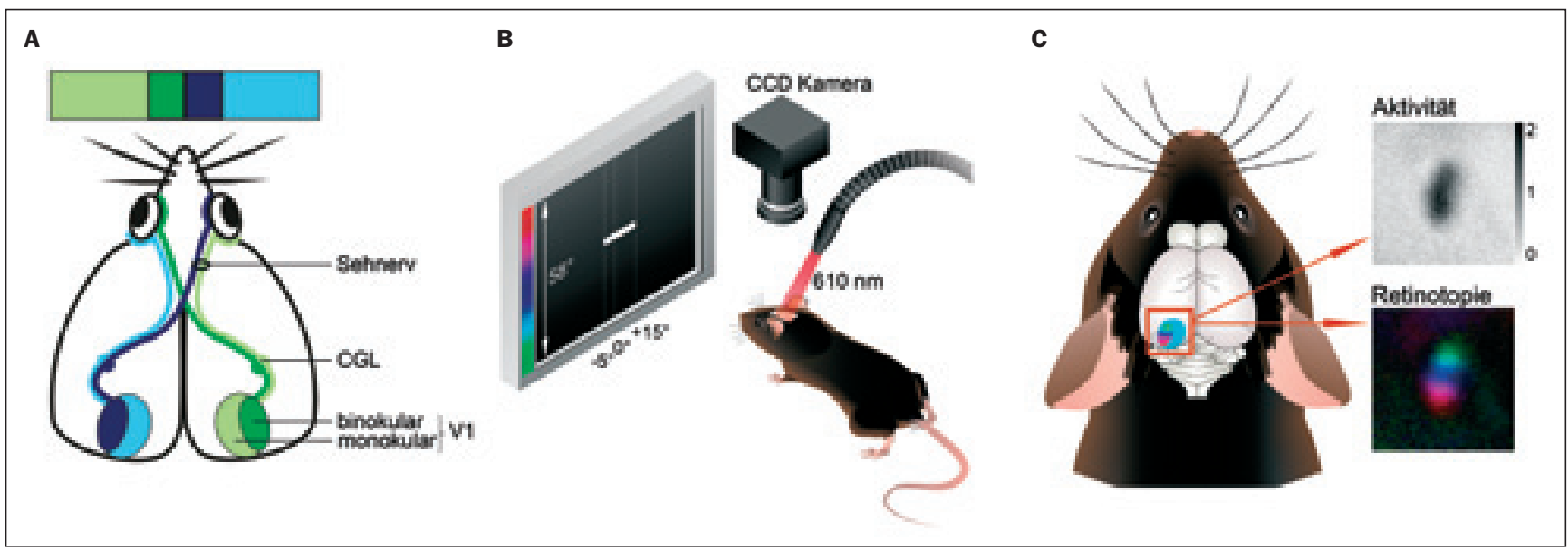

Abb. 1: Neuronale Plastizität sichtbar machen. A) Sehbahn einer Hausmaus (C57BI/6J). CGL=Corpus Geniculatum Laterale. B) Minimalinvasives optisches Ableiten neuronaler Aktivität. Das Gehirn einer Maus wird mit rotem Licht (610 nm) beleuchtet. Bei dieser Wellenlänge ist der Reflektionsunterschied von aktivem und inaktivem Gewebe besonders hoch. Auf einem Monitor wird ein sich nach oben oder unten bewegender weißer Balken auf schwarzem Hintergrund gezeigt, der die binokulare Zone der primären Sehrinde (V1) aktiviert. Die Aktivitätsänderungen in V1 werden mit einer lichtempfindlichen CCD-Kamera aufgenommen und durch Fourieranalyse (Kalatsky und Stryker 2003) sichtbar gemacht. C) Aktivitäts- (oben) und retinotope (unten) Karte im binokularen Teil von V1. Die farbkodierte retinotope Karte spiegelt die neuronale Antwort in Abhängigkeit von der räumlichen Lage des visuellen Reizes im Gesichtsfeld wider.

Form neuronaler Plastizität, die wir im Detail untersuchen (als aktuellen Übersichtsartikel empfehlen wir Levelt und Hübener 2012).

Über viele Jahre war die Standardmethode zur Bestimmung der Augendominanzplastizität die elektrophysiologische Registrierung von Aktionspotenzialen einzelner Nervenzellen in der Sehrinde (Dräger 1978; Gordon und Stryker 1996; Fagiolini et al. 2004). Damit kann zwar eine hohe räumliche Auflösung erreicht werden, es sind jedoch technisch aufwendige und langwierige Messungen nötig. Viel schneller und mit besserem Signal-Rausch-Verhältnis lässt sich die Aktivität der Sehrinde mithilfe eines minimal-invasiven optischen Verfahrens darstellen (Optical Imaging of Intrinsic Signals: zum Beispiel Grinvald et al. 1986; Kalatsky und Stryker 2003), das wir für die Analyse der Augendominanzplastizität adaptiert haben (Cang et al. 2005). Hierfür wird die Hirnoberfläche mit rotem Licht beleuchtet: Aktive Hirnrindenregionen absorbieren das Licht stärker als inaktive und können damit sogar durch den intakten Schädel mit einer sehr lichtempfindlichen CCD-Kamera als dunkle Regionen detektiert werden (Abbildung 1). Der Hauptgrund hierfür liegt vor allem in der höheren Lichtabsorption von Desoxyhämoglobin, das sich aufgrund des Sauerstoffverbrauchs aktiven Hirngewebes dort kurzfristig anreichert (Pouratian und Toga 2002). Mit diesem optischen Verfahren lassen sich erfahrungs-, lern- und altersabhängige Änderungen der Hirnaktivität nicht nur in einer Versuchsserie mit einer deprivierten und einer nicht-deprivierten
Gruppe beobachten, sondern es ist möglich, diese Änderungen an ein und demselben Tier in vivo (lateinisch ,im Lebendigen“) und über die Zeit zu verfolgen. Solch ein „chronisches" Experiment ist in Abbildung 2A-C dargestellt: Vor der MD ist der durch Reizung des kontralateralen Auges induzierte Aktivitätsfleck in V1 dunkler als der des ipsilateralen Auges, V1 wurde also stärker durch das kontralaterale Auge aktiviert. Nach der MD kommt es zu einer Augendominanzverschiebung: in unserem Beispiel ist der Aktivitätsfleck des ipsilateralen (nicht-deprivierten) Auges jetzt sogar dunkler als der des kontralateralen. Als quantitatives Maß für diese Augendominanzplastizität vergleichen wir, wie stark V1 vor und nach MD durch Reizung der beiden Augen aktiviert wird und berechnen daraus einen Augendominanzindex (ocular dominance index=ODI; Cang et al. 2005): Bei gesunden C57B1/6J-Mäusen sinkt der ODI von positiven Werten vor einer MD auf Werte nahe der 0 nach MD, was einer gleich starken Aktivierung von V1 durch beide Augen entspricht, die Augendominanz verschiebt sich also klar in Richtung des offenen Auges (Abbildung 2D).

\section{Sensorisches Lernen - das optomotorische System}

Obwohl das Sehsystem nicht das wichtigste Sinnessystem für das Überleben von Mäusen darstellt, können Mäuse ihren Sehsinn benutzen, um sich im Raum zu orientieren (Prusky et al. 2000). Vor Kurzem wurde nachgewiesen, dass monokulare Deprivation bei Mäusen und anschließendes tägliches „Training“ in einer Verhaltensapparatur, mit der das Sehvermögen mithilfe der optomotorischen Reaktion bestimmt werden kann, $\mathrm{zu}$ einer Verbesserung der Sehleistung in diesem Test führt (Prusky et al. 2006): Die Sehschärfe des offenen (nicht-deprivierten) Auges steigt bei täglichem Training um circa 25-30\% an (Abbildung 2E, F). Der Anstieg erfolgt bei wiederholtem Training schneller und hält länger an, wenn die Tiere zwei Mal pro Tag getestet werden (Prusky et al. 2006). Diese Form ,sensorischen Lernens“ weist damit wesentliche Merkmale klassischer Lernparadigmen auf. Im Gegensatz zu Verhaltenstests, die auf positiver Verstärkung beruhen und erst aufwendig von den Tieren erlernt werden müssen, wie beispielsweise dem ,visual water task“ (Gianfranceschi et al. 1999; Prusky et al. 2000; Prusky und Douglas 2003), benötigt der beschriebene Sehtest keinerlei Trainingszeit, da er auf einem Reflex, der optomotorischen Reaktion, auf einen bewegten visuellen Reiz, beruht. Solange Mäuse die bewegten senkrechten Streifenmuster sehen, folgen sie ihnen mit Kopf- beziehungsweise Oberkörperbewegungen, die vom Experimentator über eine am Deckel der Apparatur eingebaute Kamera beobachtet werden können. Während mechanische Streifenzylinder (Drehtrommeln) schon seit Langem benutzt wurden, besteht die Besonderheit der von Prusky et al. (2004) entwickelten Apparatur darin, dass vier um die Maus positionierte Flachmonitore einen virtuellen Zylinder erzeugen, der auf die 


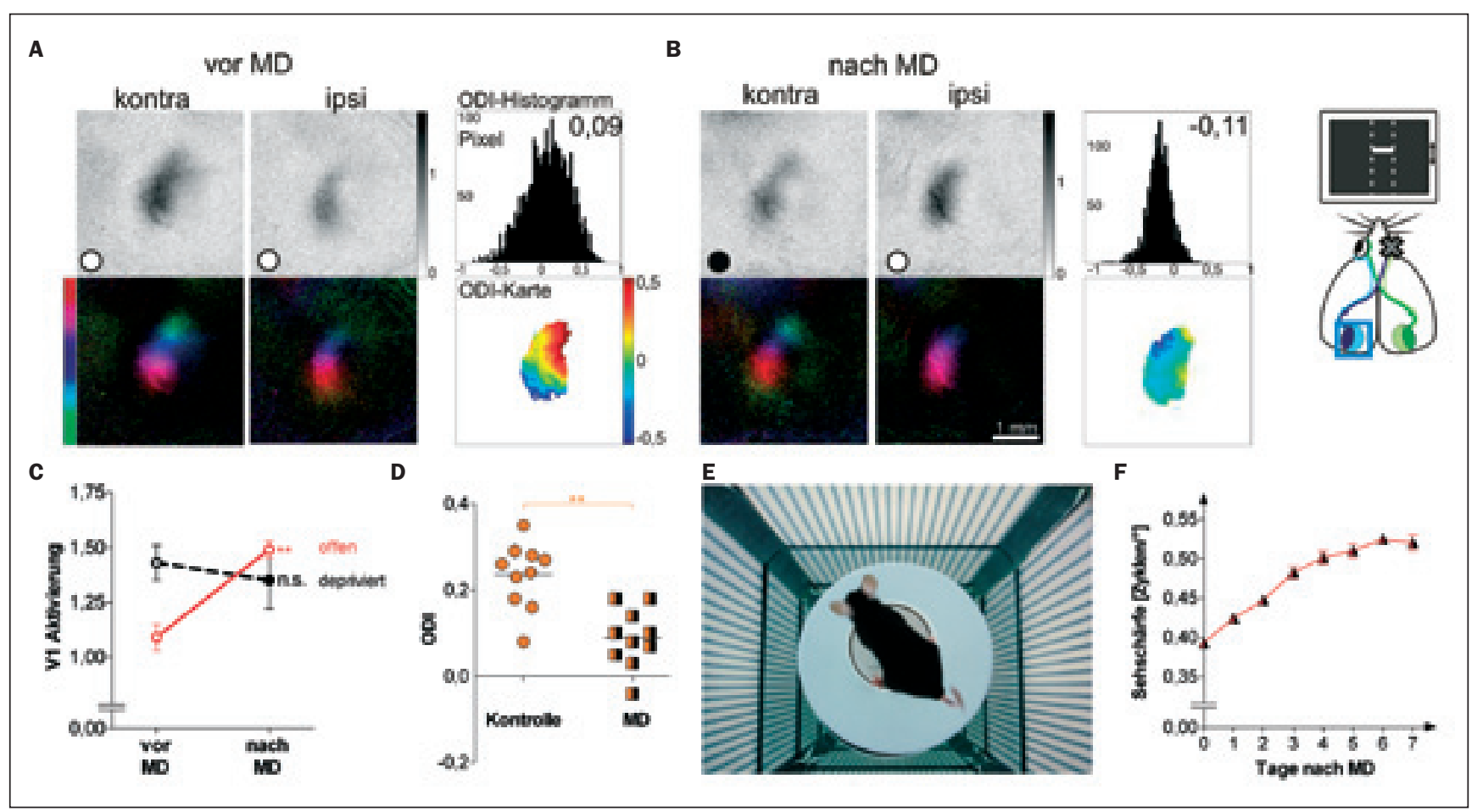

Abb. 2: Zwei Modelle erfahrungsabhängiger Plastizität im Sehsystem von Mäusen: Augendominanzplastizität (A-D) und sensorisches Lernen (E, F) nach monokularer Deprivation (MD). A-C) Aktivierung der primären Sehrinde (V1) nach Reizung des kontra- beziehungsweise ipsilateralen Auges vor (A) und nach MD (B) und deren Quantifizierung (C) beim gleichen Tier. Gezeigt sind grauwertkodierte Aktivitäts(oben) und farbkodierte retinotope Karten (unten) aus dem binokularen Teil der linken Sehrinde (A, B). Die Stärke der Aktivierung ist als Änderung in der Reflektion $\left(\times 10^{4}\right)$ dargestellt. Vor MD ist der durch Reizung des kontralateralen Auges induzierte Aktivitätsfleck dunkler als der des ipsilateralen Auges, der Augendominanzindex (ODI) ist positiv und die ODI-Karte vor allem gelb-rot (rot repräsentiert positive, blau negative ODI-Werte), das heißt, das kontralaterale Auge dominiert die V1-Aktivität (A). Nach sieben Tagen MD des kontralateralen (rechten) Auges kommt es zu einer Augendominanzverschiebung in Richtung des offenen (ipsilateralen) Auges: Das offene Auge aktiviert die Sehrinde jetzt stärker, der ODI ist verringert und die ODI-Karte weist kühlere Farben auf (B, C). D) Änderung der ODIs nach MD: Werte fallen von positiven (kontralaterale Dominanz) auf Werte um 0 ab, das heißt, beide Augen aktivieren V1 jetzt ähnlich stark. E, F) Tägliches Training im optomotorischen System (E) führt zu einer deutlichen Erhöhung der Sehschärfe des offenen Auges nach MD (F). D, F) verändert nach Lehmann und Löwel 2008.

Augenposition der Maus zentriert werden kann. Dadurch kann die Ortsfrequenz der präsentierten Streifenmuster immer exakt festgelegt werden, auch wenn sich die Maus bewegt. Es soll hier nicht unerwähnt bleiben, dass die optomotorisch gemessene „Sehschärfe" nicht der maximalen Sehschärfe der Tiere entspricht. Die gemessenen Werte stehen jedoch in einer relativ konstanten $\mathrm{Be}$ ziehung zur maximalen Sehschärfe, die man beispielsweise mit visuell evozierten Potenzialen, mit optischem Ableiten oder mit dem „visual water task" messen kann (Porciatti et al. 1999; Prusky und Douglas 2003; Goetze et al. 2010), sodass sie als valides Maß für die Leistungsfähigkeit des Sehens der Mäuse angesehen werden können. Während die optomotorische Reaktion als solche auf einem Reflex beruht, der über Netzwerke außerhalb des Kortex (subkortikal) vermittelt wird, beruht die Sehschärfezunahme nach einer MD auf Veränderungen in der Hirnrinde (Prusky et al. 2006).

\section{1x Maus - 2x Plastizität}

Kombiniert man die beiden Analysetechniken - das optische Ableiten intrinsischer Signale und die Messung des Sehvermögens mit dem optomotorischen System - kann man zwei Modelle erfahrungs- beziehungsweise lernabhängiger Plastizität in ein und demselben Tier untersuchen: Die Augendominanzplastizität als etabliertes Modell kortikaler Plastizität und zusätzlich die Zunahme der Sehschärfe des offenen Auges nach MD als Modell sensorischen Lernens im Verhaltensversuch.

\section{Verändern sich Augendominanz-} plastizität und sensorisches Lernen im Alter und nach Läsionen?

OD-Plastizität ist altersabhängig (Abbildung 3). Augendominanzplastizität lässt sich bei Mäusen in der sogenannten kritischen Phase, also einem Alter von
19-32 Tagen, mit nur vier Tagen monokularer Deprivation (MD) auslösen (Gordon und Stryker 1996; Lehmann und Löwel 2008). Mit zunehmendem Alter nimmt die Plastizität jedoch ab und es bedarf einer längeren MD-Dauer (sieben Tage), um Augendominanzplastizität herbeizuführen (Sawtell et al. 2003; Lehmann und Löwel 2008; Sato und Stryker 2008). Zudem fällt auf, dass der Augendominanzindex (ODI) weniger stark absinkt, die Veränderung der Augendominanz also weniger stark als bei jüngeren Tieren ausgeprägt ist. $\mathrm{Ab}$ einem Alter von 110 Tagen lässt sich schließlich überhaupt keine Augendominanzplastizität mehr auslösen (Lehmann und Löwel 2008), auch nicht nach 14 Tagen MD. Welche molekularen und zellulären Veränderungen diesem Prozess zugrunde liegen und wie die Plastizität im alternden Gehirn wieder erhöht werden kann, ist Gegenstand intensivster Forschungen und natürlich auch für Fragen der Regenerati- 
on nach Läsionen wichtig (Bavelier et al. 2010; Smith und Bear 2010; Baroncelli et al. 2011)

Sensorisches Lernen ist altersabhängig (Abbildung 4). Interessanterweise zeigt auch das sensorische Lernen nach MD bei Mäusen eine ausgeprägte Altersabhängigkeit (Lehmann und Löwel 2008; Lehmann et al. 2012). Es ist bei 1-3 Monate alten Tieren signifikant höher als bei 4-7 Monate alten Tieren und sinkt bis zum Alter von knapp zwei Jahren noch weiter ab: Die Sehschärfezunahme ist im Vergleich zu vier oder sieben Monate alten Mäusen um mehr als die Hälfte verringert (Abbildung 4A, B). Überdies wird die Sehschärfezunahme mit dem Alter bei einzelnen Tieren immer variabler (Abbildung 4C-F): Im Alter von 23 Monaten, also zirka zwei Jahren, zeigten einzelne Mäuse nach MD eine ebenso große Sehschärfezunahme wie jüngere Tiere, während andere nur eine geringe oder gar keine Sehschärfezunahme aufweisen. Die interindividuelle Variabilität des sensorischen Lernvermögens steigt also mit zunehmendem Alter dramatisch an. Woran das liegt und wie man bei Älteren Lernleistungen fördern kann, ist eine besonders spannende wissenschaftliche Fragestellung. Die zunehmende Variabilität bei alten Mäusen legt nahe, dass die abnehmende sensorische Plastizität nicht nur durch altersabhängige Prozesse bedingt ist, die bei allen Tieren ähnlich ablaufen, sondern auch durch individuelle Begebenheiten und Umwelteinflüsse. Kürzlich wurde gezeigt, dass im alternden Gehirn zunehmend entzündliche Prozesse stattfinden (Sparkman und Johnson 2008) und die durch Lernen induzierte Histon-Acetylierung gestört ist (Stilling und Fischer 2011). Beide Effekte können individuell unterschiedlich sein, und sie sind wahrscheinlich zudem abhängig von individuellen Umweltbedingungen, Ernährung, Stress und dem allgemeinem Gesundheitszustand.

\section{Ein Schlaganfall beeinträchtigt sowohl kortikale Plastizität als auch sensorisches Lernen in weit entfernt liegenden Hirnregionen}

Ein praktisches Beispiel für die erfolgreiche Kombination der beiden beschriebenen Plastizitätsmodelle im gleichen Tier ist unsere kürzlich erschienene Studie zum Einfluss eines Schlaganfalls auf neuronale Plastizität (Greifzu et al. 2011). Wir konnten zeigen, dass ein lokaler Schlaganfall selbst entfernt liegende Hirnbereiche, die ursprünglich gar nicht betroffen sind, be- einträchtigen kann. Wenn die Blutzufuhr zum Gehirn durch eine verstopfte Ader oder ein geplatztes Gefäß unterbrochen wird, bleiben auch der Sauerstoff und die Nährstoffe aus. Nervenzellen im betroffenen Areal sterben und Körperteile, die von diesen Zellen gesteuert werden, können nicht mehr richtig funktionieren. Ein Schlaganfall ist die dritthäufigste Todesursache in Deutschland. Aber auch ein Großteil derer, die gerettet werden können, leidet unter bleibenden Schäden. Einige davon können durch entsprechende Rehabilitationsmaßnahmen wieder behoben oder zumindest vermindert werden. So müssen oft einfachste Tätigkeiten wie Gehen oder Sprechen neu erlernt werden.

In unserer Studie wurde bei drei Monate alten Mäusen in einem lokalen Areal außerhalb der Sehrinde ein Schlaganfall ausgelöst. Als Schlaganfallmodel nutzten wir die Photothrombosetechnik von Watson et al. (1985). In unserem Fall wurde die Läsion im primären somatosensorischen Kortex positioniert (Abbildung 5). Anschließend untersuchten wir die Plastizität im Sehsystem von Mäusen in vivo. Verschließt man bei Kontrolltieren ohne Schlaganfall für sieben Tage ein Auge, erhöht sich die Sehschärfe des offenen Auges und die Augendominanz verschiebt sich hin zum offenen Auge (Abbildung 2). Nach einem Schlaganfall blieb diese Plastizität jedoch vollständig aus (Abbildung 6): V1 war weiterhin dominiert vom kontralateralen (geschlossenen) Auge, wie vor MD und die ODIs veränderten sich nicht. Auch stieg die Sehschärfe des offenen Auges trotz täglichen Trainings der Tiere nicht an. Es gab also nach dem Schlaganfall weder eine signifikante Verschiebung der Augendominanz noch sensorisches Lernen. Das Ausbleiben des Sehschärfeanstiegs war besonders überraschend, da Untersuchungen von Glen Prusky und seinen Kollegen (Prusky et al. 2006) gezeigt hatten, dass für die Verbesserung der Sehschärfe nach MD die Hirnhälfte kontralateral zum offenen Auge benötigt wird. Dies war in unserem Fall jedoch die dem Schlaganfall gegenüberliegende Seite, die gar nicht verletzt wurde. Was lernen wir daraus? Es gibt offenbar keine ,intakte“ Hemisphäre, da es selbst nach einer kleinen und lokalen Verletzung im Gehirn Auswirkungen auf Nervennetzwerke beider Hirnhälften gibt (Andrews 1991). Auch die verloren gegangene Augendominanzplastizität deutet auf einen klaren Einfluss weitreichender Netzwerke auf neuronale Plastizität hin: Sie zeigt, dass eine Änderung der Aktivität in den Hauptverbindungen von den Augen über den Thalamus zur Sehrinde nicht ausreicht, um dort Augendominanzplastizität auszulösen und sich die Sensibilität der Hirnrinde für Änderungen afferenter Aktivität offenbar modulieren lässt. Augendominanzplastizität ist demzufolge nicht nur ein lokales Phänomen, sondern wird vielmehr durch Netzwerkeinflüsse auch von außerhalb der Sehrinde und unabhängig von den thalamo-kortikalen

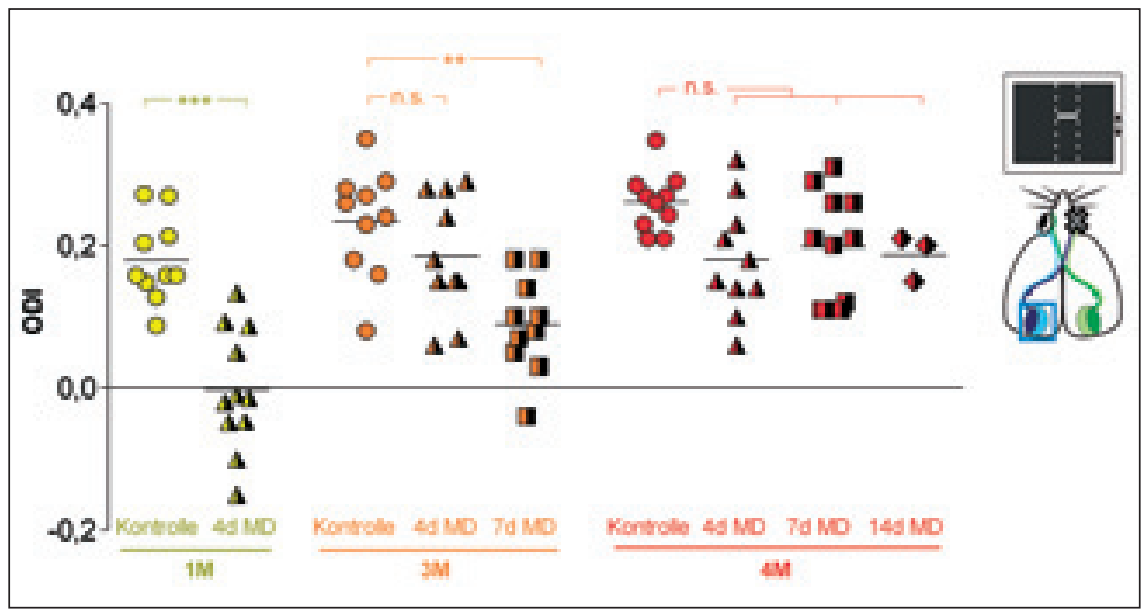

Abb. 3: Augendominanzplastizität nimmt mit zunehmendem Alter ab. Gezeigt ist der Augendominanzindex (ODI) verschiedener Altersgruppen von Mäusen, vor (Kontrolle) und nach MD. Symbole repräsentieren ODI-Werte einzelner Tiere, waagerechte Striche den Mittelwert. Bei einem Monat (1M) alten Mäusen reichen vier Tage MD (4d MD), bei drei Monate (3M) alten Tieren werden sieben Tage MD für eine signifikante Augendominanzverschiebung benötigt. Ab einem Alter von 110 Tagen (4M) lässt sich überhaupt keine Augendominanzplastizität mehr auslösen, auch nicht nach 14 Tagen MD (verändert nach Lehmann und Löwel 2008). 
Verbindungen mitbestimmt. Dies erinnert an ältere Untersuchungen zum Einfluss modulatorischer Systeme, wie zum Beispiel der cholinergen und noradrenergen, auf die Plastizität in der Sehrinde von jungen Katzen (Bear und Singer 1986). Wenn man die modulatorischen Afferenzen in die Hirnrinde unterband, wirkte sich eine MD kaum auf die binokularen Verschaltungen der primären Sehrinde aus: Die Plastizität wird also auch von extraretinalen Faktoren mitbestimmt.

\section{Weitreichende Wechselwirkungen kontrollieren Plastizitätsprozesse auch im gesunden Gehirn}

Eine Reihe weiterer Forschungsergebnisse stützen unsere Interpretation eines Einflusses weitreichender Netzwerke auf die neuronale Plastizität. So zeigen neuere Untersuchungen, dass die Augendominanzplastizität bei Ratten maßgeblich von Verbindungen zwischen den beiden Hirnhälften über den Balken (Corpus callosum; lateinisch corpus „Körper“, callus „Schwiele“) abhängt (Restani et al.
2009). Dabei werden vor allem Eingänge vom ipsilateralen Auge über das Corpus callosum vermittelt, die eine entscheidende Rolle bei der Reduzierung der Eingänge vom deprivierten Auge während der MD spielen. Bei unseren Schlaganfall-Experimenten sollten die callosalen Eingänge aus der nicht-läsionierten Hemisphäre allerdings intakt sein und dennoch gab es keine Augendominanzplastizität. Ob diese Ergebnisse auf eine geringere Bedeutung der callosalen Verbindungen bei Mäusen (im Vergleich zur Ratte) schließen lassen, ist noch nicht bekannt.

Auch im Gehirn größerer Säugetiere haben wir deutliche Hinweise darauf gefunden, dass Plastizitätsprozesse von weitreichenden Wechselwirkungen kontrolliert werden. Bei Katzen und Primaten liegen Neurone mit gleichen funktionellen Eigenschaften nicht ungeordnet in der Hirnrinde verteilt, sondern bilden regelmäßige Muster, sogenannte „Karten“: Die Anordnung von Neuronen gleicher Augendominanz (Augendominanzkarte) oder gleicher bevorzugter Kantenorientierung (Orientierungskarte) lässt sich durch metabolische oder hämodynamische Bildgebungsverfahren in großen Bereichen von V1 sichtbar machen. In Untersuchungen der geometrischen Eigenschaften dieser Karten bei Katzen verschiedener Altersgruppen konnten wir nachweisen, dass sich beide Muster im Verlauf der kritischen Phase reorganisieren. So erhöht sich die Anzahl der von jeweils einem Auge dominierten Bereiche in der Sehrinde von Katzen zwischen der vierten und zehnten Lebenswoche um mehr als 20\% (Keil et al. 2010). Auch die Orientierungskarte gestaltet sich während dieser späten Phase der kritischen Periode um (Kaschube et al. 2009). Durch den Vergleich von Kartenmustern in beiden Gehirnhälften und in verschiedenen Arealen der Sehrinde fanden wir, dass der Reorganisationsprozess in beiden Hemisphären koordiniert verläuft: Die Größe der Orientierungsdomänen in miteinander verbundenen Hirngebieten wird hierbei systematisch aneinander angepasst (Kaschube et al. 2009).

Theoretische Untersuchen sagten schon länger vorher, dass weitreichende Verbindungen auch innerhalb der Sehrinde
A

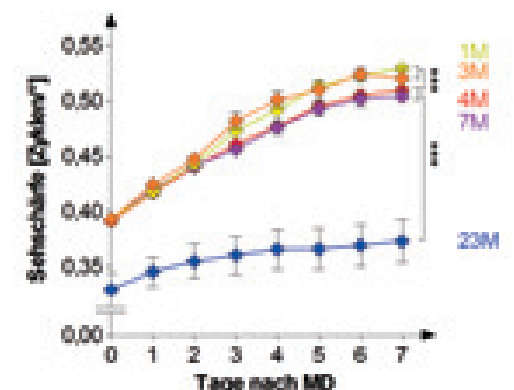

C

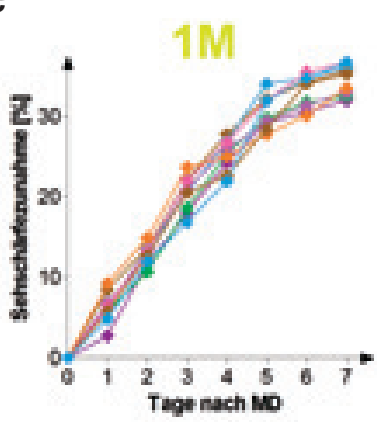

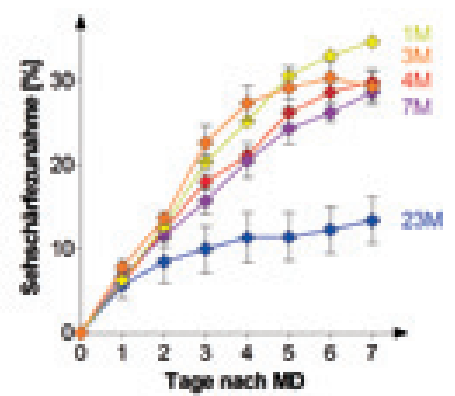

E

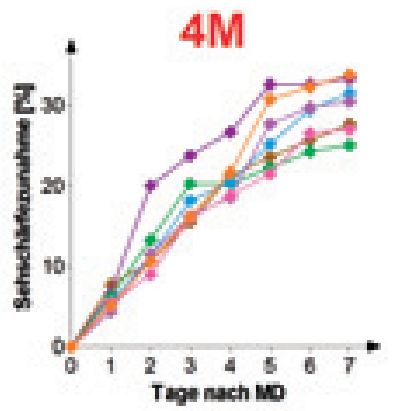

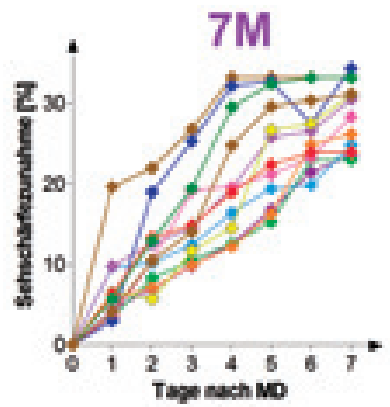

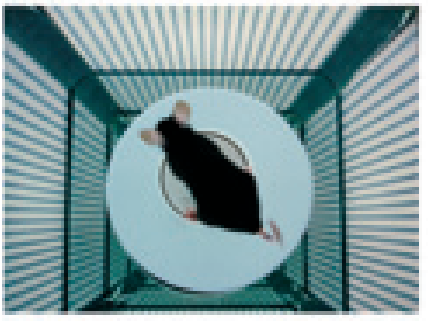

$\mathbf{F}$

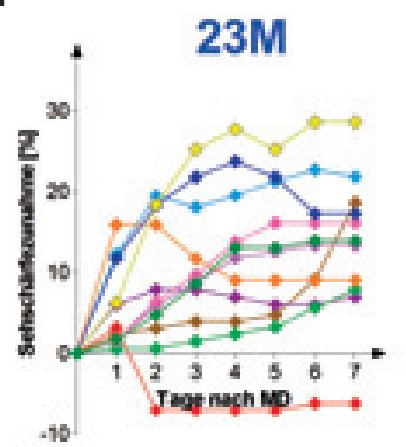

Abb. 4: Sensorisches Lernen nimmt mit dem Alter ab. Mäuse verschiedener Altersstufen (1, 3, 4, 7 und 23 Monate (M)) wurden monokular depriviert (MD) und täglich im optomotorischen System getestet. Aufgetragen ist die Sehschärfe (Zyklen pro Sehwinkelgrad, (A)), beziehungsweise die prozentuale Sehschärfezunahme nach MD (B-F). Die Sehschärfe des offenen Auges verbesserte sich bei allen untersuchten Altersgruppen signifikant, allerdings ist diese Zunahme bei 23 Monate alten Mäusen um mehr als die Hälfte verringert im Vergleich zu vier oder sieben Monate alten Tieren. Besonders auffallend ist, dass die Sehschärfezunahme mit steigendem Alter immer variabler wird: Bei 23M alten Mäusen zeigten einige Tiere sensorisches Lernen wie vier Monate alte Tiere, während andere keine oder eine nur sehr geringe Zunahme zeigten (verändert nach Lehmann und Löwel 2008; Lehmann et al. 2012). 


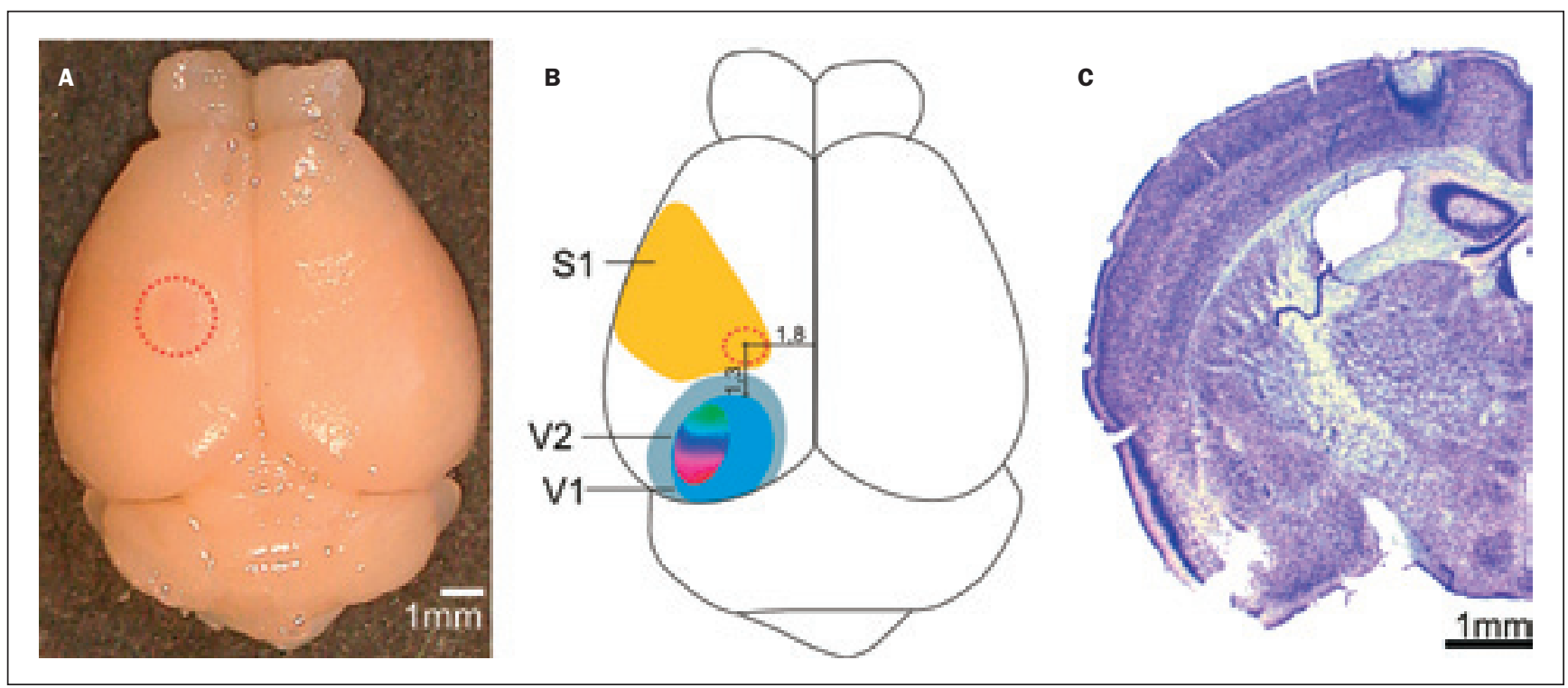

Abb. 5: Lage und Größe der photothrombotischen (PT) Läsion in der Hirnrinde der Maus. A) Blick von oben auf ein Mäusegehirn mit einer PT-Läsion in der linken Hemisphäre (roter gepunkteter Kreis). B) Das Zentrum der PT-Läsion befand sich im primären somatosensorischen Kortex (S1), im Durchschnitt 1,3 mm anterior zur anterioren Grenze der primären Sehrinde (V1) und 1,8 mm lateral von der Mittellinie. Eine optisch abgeleitete retinotope Karte aus dem binokularen Teil von V1 zeigt die Lage des von uns untersuchten Areals im Vergleich zur Läsion. C) Im NissI-gefärbten Frontalschnitt vom Mäusegehirn ist die Läsion im linken S1 deutlich sichtbar (verändert nach Greifzu et al. 2011).

entscheidend zur Formung und Stabilisierung der räumlichen Anordnung der Orientierungsdomänen beitragen (Wolf 2005; Kaschube et al. 2008). Die mathematisch vorhergesagten Kennzeichen weitreichender Wechselwirkungen im Muster der Orientierungskolumen konnten wir mit überraschender Klarheit in der Sehrinde von Katzen, Frettchen, Spitzhörnchen und Buschbabies nachweisen (Kaschube et al. 2010; Keil et al. 2012). In all diesen Tierarten ist bekannt, dass die Sehrinde von einem System weitreichender axonaler Verbindungen durchzogen wird. Diese neuronale Architektur scheint auszureichen, den Mustern eine artunabhängige Geometrie aufzuprägen, die sich nur bei Berücksichtigung weitreichender Verbindungsstrukturen in Computermodellen der kortikalen Plastizität nachbilden lässt. Ergebnisse wie diese lassen erwarten, dass die Aufklärung der Natur einer weitreichenden Modulation neuronaler Plastizität nach Schlaganfall auch einen Blick auf allgemeine Mechanismen der Plastizität und Stabilisierung von Netzwerken der Großhirnrinde eröffnen kann.

\section{Therapieansätze}

Da ein Schlaganfall mit einer Entzündungsreaktion einhergeht (Garcia 1975), untersuchten wir auch, ob diese für die beobachteten Störungen verantwortlich sein könnte. Dazu behandelten wir die
Tiere direkt nach dem Schlaganfall einmal täglich mit dem entzündungshemmenden Medikament Ibuprofen. Tatsächlich konnten wir damit die gestörte sensorische Lernfähigkeit wieder komplett herstellen - sowohl Sehschärfe als auch Kontrastsensitivität des offenen Auges nahmen sogar schon am ersten Tag nach Behandlung wieder stark zu und erreichten Werte wie bei Kontrolltieren (Abbildung 6D). Hatte das Gehirn nach dem Schlaganfall zwei Wochen Zeit, sich zu erholen, war das sensorische Lernen nach MD ebenfalls wiederhergestellt (Greifzu et al. 2011). Dieser Befund unterstützt unsere Hypothese, dass Entzündungsvorgänge das sensorische Lernen beeinflussen können, da Entzündungsreaktionen nach einem Schlaganfall normalerweise innerhalb einer Woche wieder zurückgehen (Liu et al. 1993; Wang et al. 1994). Unsere Untersuchungen weisen deshalb darauf hin, dass Entzündungsreaktionen direkten Einfluss nehmen können auf neuronale Aktivitätsmuster, die essenziell für sensorische Lernvorgänge im $\mathrm{Ge}$ hirn sind. Und dieser hemmende Einfluss ist nicht-lokal, da Entzündungsfaktoren über den Blutfluss im gesamten Gehirn (und Körper) verteilt werden können. Es spricht somit einiges dafür, dass die Gabe entzündungshemmender Substanzen eine nützliche Zusatztherapie für die Förderung der Rehabilitation nach einem Schlaganfall darstellt. Im Gegensatz zum sensorischen Lernen konnte die Augen- dominanzplastizität jedoch weder durch eine entzündungshemmende Behandlung noch durch eine Verzögerung der MD nach dem Schlaganfall wiederhergestellt werden (Abbildung 6C). Dies zeigt, dass diese zwei Modelle visueller Plastizität über unterschiedliche Mechanismen und neuronale Schaltkreise vermittelt werden. Laut Untersuchungen von Prusky et al. (2006) ist die Zunahme der Sehschärfe des offenen Auges nach MD auf die monokulare Region in der Sehrinde beschränkt, während die Verschiebung der Augendominanz den Wettbewerb der Afferenzen der beiden Augen um kortikales Territorium darstellt und damit in der binokularen Region der primären Sehrinde stattfindet (Hubel et al. 1977; Gordon und Stryker 1996; Levelt und Hübener 2012).

\section{Fazit}

Dem Gehirn beim Lernen zusehen: dieser lange gehegte Traum von Neurowissenschaftlern ist in greifbare Nähe gerückt. Die hier beschriebenen Untersuchungsmethoden sind hervorragend dafür geeignet, Verhaltensuntersuchungen mit der Sichtbarmachung neuronaler Aktivitätsmuster beim gleichen Tier zu kombinieren: Wir können damit erfahrungs-, lern- und altersabhängige Änderungen der Hirnaktivität direkt am lebenden Tier, also in vivo, und sogar über längere Zeiträume beobachten. Durch die Möglichkeit, auch genetisch 


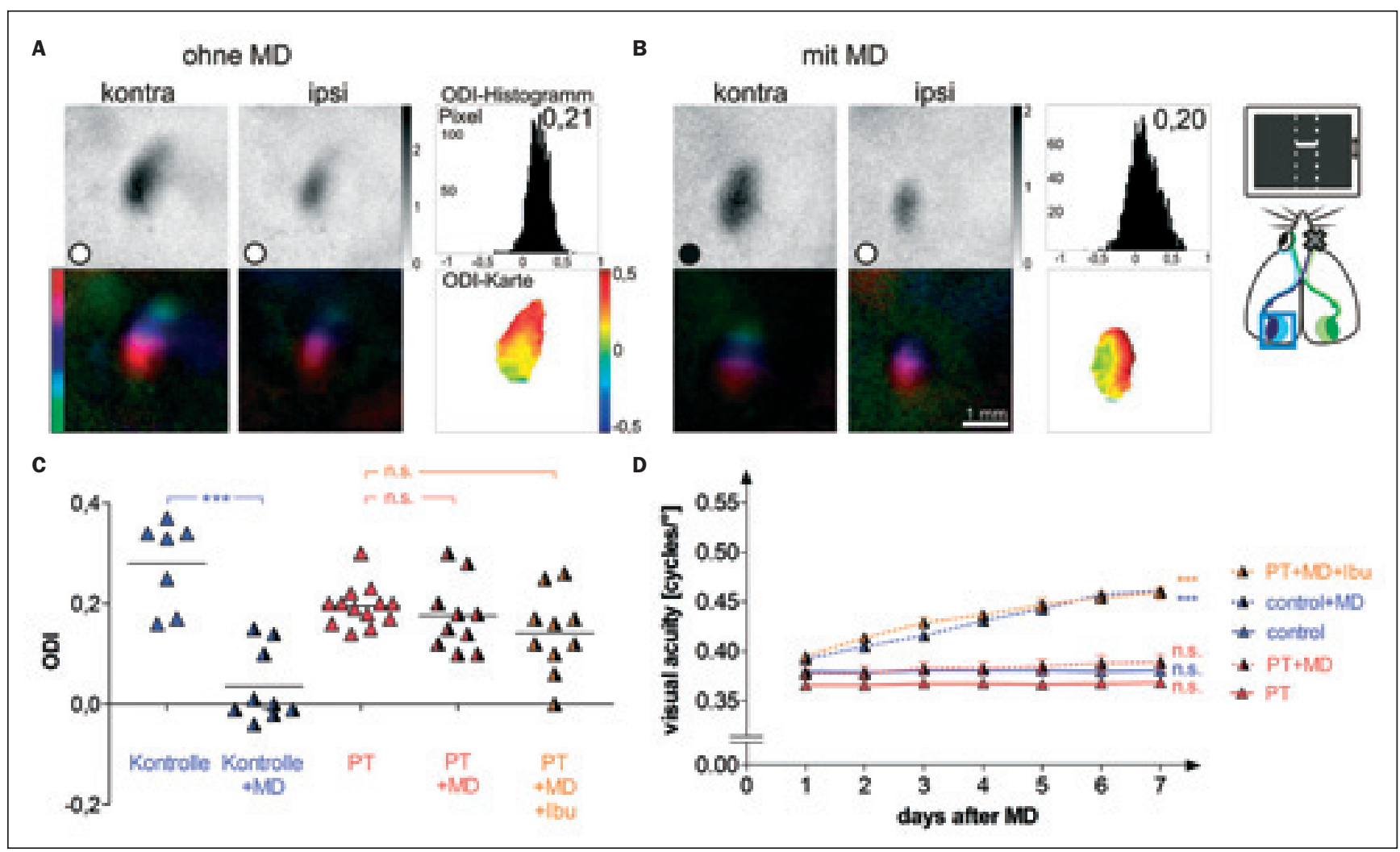

Abb. 6: Ein lokaler Schlaganfall schädigt Plastizität und Lernvermögen auch in entfernt liegenden Gehirnregionen. Eine entzündungshemmende Therapie ist zum Teil wirksam. A-C) Optisch registrierte Aktivierung der Sehrinde nach Reizung des kontra- beziehungsweise ipsilateralen Auges ohne (A) und mit MD (B) bei Mäusen mit einer photothrombotischen Läsion (PT) in S1. Darstellung wie in Abbildung 2 und 3. Im Gegensatz zu Kontrolltieren (Abbildung 2) löst eine MD bei Tieren mit einem Schlaganfall keine signifikante Augendominanzverschiebung aus: V1 wird weiterhin stärker vom kontralateralen (geschlossenen!) Auge aktiviert, ODI-Werte sind unverändert und warme Farben überwiegen in der ODI-Karte. D) Eine PT-Läsion verhindert auch die Sehschärfezunahme nach MD. Aufgetragen ist die Sehschärfe für die jeweilige Gruppe über sieben Tage. Bei Kontrolltieren steigt die Sehschärfe des offenen Auges während der MD signifikant an (Kontrolle+MD). Bei PT-Tieren mit einer Läsion blieb dieser Effekt jedoch aus (PT+MD). Behandelt man die Tiere mit der entzündungshemmenden Substanz Ibuprofen (PT+MD+lbu), so wird das sensorische Lernen vollständig wiederhergestellt. Die Augendominanzplastizität konnte jedoch nicht therapiert werden (C) (verändert nach Greifzu et al. 2011).

modifizierte Mäuse zu analysieren, können zudem ganz neue Einblicke in zelluläre und molekulare Mechanismen neuronaler Plastizität gewonnen und die Veränderungen von Aktivitätsmustern im Gehirn direkt mit den Veränderungen des Verhaltens verglichen werden (Goetze et al. 2010; Krempler et al. 2011; Greifzu et al. 2011).

\section{Literatur}

Cang, J., Kalatsky, V.A., Löwel, S. und Stryker, M.P. (2005): Optical imaging of the intrinsic signal as a measure of cortical plasticity in the mouse. Vis Neurosci 22: 685-691.

Gordon, J.A. und Stryker, M.P. (1996): Experience-dependent plasticity of binocular responses in the primary visual cortex of the mouse. J Neurosci 16: 3274-3286.

Greifzu, F., Schmidt, S., Schmidt, K.-F., Kreikemeier, K., Witte, O.W. und Löwel, S. (2011): Global impairment and therapeutic restoration of visual plasticity mechanisms after a loca- lized cortical stroke. Proc Natl Acad Sci USA 108: 15450-15455.

Kalatsky, V.A. und Stryker, M.P. (2003): New paradigm for optical imaging: temporally encoded maps of intrinsic signal. Neuron 38: 529-545.

Kaschube, M., Schnabel, M., Wolf, F. und Löwel, S. (2009): Interareal coordination of columnar architectures during visual cortical development. Proc Natl Acad Sci USA 106: 17205-17210.

Kaschube, M., Schnabel, M., Löwel, S., Coppola, D.M., White, L.E. und Wolf, F. (2010): Universality in the evolution of orientation columns in the visual cortex. Science 330: 1113-1116.

Keil, W., Schmidt, K.-F., Löwel, S. und Kaschube, M. (2010): Reorganization of columnar architecture in the growing visual cortex. Proc Natl Acad Sci USA 107: 12293-12298.

Lehmann, K. und Löwel, S. (2008): Age-dependent ocular dominance plasticity in adult mice PLOS ONE 3(9): e3120.

Lehmann, K., Schmidt, K.-F. und Löwel, S. (2012): Vision and visual plasticity in ageing mice. Restor Neurol Neurosci 30: 161-178.
Levelt, C.N. und Hübener, M. (2012): Criticalperiod plasticity in the visual cortex. Annu Rev Neurosci 35: 309-330.

Prusky, G.T., Alam, N.M. und Douglas, R.M. (2006): Enhancement of vision by monocular deprivation in adult mice. $J$ Neurosci 26: 11554-11561.

Wiesel, T.N. und Hubel, D.H. (1963): Singlecell responses in striate cortex of kittens deprived of vision in one eye. J Neurophysiol 26: 1003-1017.

Eine vollständige Literaturliste kann bei den Autoren angefordert werden.

\section{Danksagung}

Die Autoren danken allen Kolleginnen und Kollegen, mit denen sie im Rahmen der hier vorgestellten Projekte zusammengearbeitet haben. Besonderer Dank geht an Prof. Dr. Hans-Joachim Bischof für hilfreiche Kommentare zum Ma- 
nuskript und an Christian Spalthoff für seine Unterstützung bei der Erstellung von Abbildung 1. Die hier vorgestellten Arbeiten wurden gefördert durch das Bundesministerium für Bildung und Forschung (BMBF) 01GQ0430, 01GQ0922, 01GQ1005, 01GQ0811，01GQ0810 und 01GQ0921, die Human Frontier Science Programm Organisation (HFSP), die Volkswagen Stiftung und die Max-PlanckGesellschaft.

\section{Kurzbiografien}

Franziska Greifzu studierte Biologie, Psychologie und Geografie an der Friedrich-Schiller-Universität Jena, sowie der Universidad Complutense in Madrid. Sie begann ihre Promotion 2009 in der Arbeitsgruppe von Prof. Dr. Siegrid Löwel an der Universität in Jena und setzt sie seit 2011 an der Georg-August-Universität in Göttingen fort, wo sie im November 2011 in die Göttinger Graduiertenschule für Neurowissenschaften, Biophysik und Molekulare Biowissenschaften (GGNB) aufgenommen wurde.

Fred Wolf studierte Physik und Neurowissenschaften in Frankfurt am Main, 1999 Promotion in theoretischer Physik (Dr. phil. nat.). Nach seiner Promotion forschte Wolf als Amos de Shalit Fellow der Minerva Stiftung an der Hebräischen Universität von Jerusalem (Israel) und an der University of California in Santa Barbara (USA) als Postdoctoral Fellow. Er leitet seit 2004 die Forschungsgruppe Theoretische Neurophysik am Max-Planck-Institut für Dynamik und Selbstorganisation in Göttingen und ist Vorstandsmitglied des
Göttinger Bernstein Zentrums für Computational Neuroscience. Seit 2008 ist Wolf Honorarprofessor der Physik an der GeorgAugust-Universität Göttingen. Im Jahr 2010 leitete er das Forschungsprogramm Emerging Techniques in Neuroscience am Kavli Institute for Theoretical Physics der University of California in Santa Barbara (USA). Wolf wurde im Jahr 1999 mit dem erstmals vergebenen Altdorfer-LeibnizPreis ausgezeichnet und war im Jahr 2011 Gastprofessor für mathematische Neurowissenschaften an der Universität von Marseilles (Frankreich).

Siegrid Löwel studierte Biologie in Würzburg und Frankfurt, 1988 Promotion (Dr. phil. nat.) und 1995 Habilitation in Zoologie an der Johann-Wolfgang-GoetheUniversität in Frankfurt am Main, bis 1996 wissenschaftliche Mitarbeiterin am Max-Planck-Institut für Hirnforschung in Frankfurt am Main in der Neurophysiologischen Abteilung von Prof. Dr. Wolf Singer, 1997-2005 Leiterin der Forschergruppe ,Visuelle Entwicklung und Plastizität" am Leibniz-Institut für Neurobiologie in Magdeburg, 2002-2003 Research Associate Professor am KeckCenter der University of California in San Francisco (USA), im Labor von Prof. Dr. Michael P. Stryker, 2003-2004 DorotheaErxleben-Gastprofessorin an der Ottovon-Guericke-Universität Magdeburg, 2004-2005 Stipendiatin im Hertie-Exzellenz-Programm „Neurowissenschaften“, 2005-2010 Universitätsprofessorin (W2) für Neurobiologie an der Friedrich-Schiller-Universität Jena, seit August 2010 Universitätsprofessorin (W3) für Systemische Neurobiologie am Bernstein Fokus Neuro- technologie und der Biologischen Fakultät der Georg-August-Universität Göttingen.

\section{Korrespondenzadressen}

\section{Franziska Greifzu}

Systems Neuroscience

Johann-Friedrich-Blumenbach-Institut für

Zoologie und Anthropologie

Bernstein Fokus Neurotechnologie

Georg-August-Universität Göttingen

Von-Siebold-Str. 4

37075 Göttingen

Tel.: $\quad+495513920163$

Fax: $\quad+495513920162$

E-Mail: franziska.greifzu@biologie.unigoettingen.de

\section{Prof. Dr. Fred Wolf}

Theoretische Neurophysik

Max-Planck-Institut für Dynamik und

Selbstorganisation

Am Faßberg 17

37077 Göttingen

Tel.: $\quad+495515176423$

Fax: $\quad+495515176409$

E-Mail:fred@nld.ds.mpg.de

\section{Prof. Dr. Siegrid Löwel}

Systems Neuroscience

Johann-Friedrich-Blumenbach-Institut für

Zoologie und Anthropologie

Bernstein Fokus Neurotechnologie

Georg-August-Universität Göttingen

Von-Siebold-Str. 4

37075 Göttingen

Tel.: $\quad+495513920161 / 60$

Fax: $\quad+495513920162$

E-Mail: sloewel@gwdg.de

(C) Springer-Verlag GmbH 2012

\section{Tierversuche in der Forschung}

Die Leopoldina - Nationale Akademie der Wissenschaften und die Union der deutschen Akademien der Wissenschaften haben eine gemeinsame ad-hoc-Stellungnahme zur Umsetzung der EU-Richtlinien zum Tierschutz in deutsches Recht herausgegeben.

Am 22. September 2010 haben das Europäische Parlament und der Rat der Europäischen Union eine Richtlinie zum Schutz der für wissenschaftliche Zwecke verwendeten Tiere erlassen. Sie zielt im Wesentlichen auf eine Harmonisierung der stark divergierenden Vorschriften der Mitgliedstaaten, in denen sie bis zum 10. November 2012 in nationales Recht umgesetzt werden muss. In Deutschland liegt dazu seit Kurzem ein Gesetz- und Verordnungsentwurf aus dem Bundesministerium für Ernährung, Landwirtschaft und Verbraucherschutz vor.

Die Nationale Akademie der Wissenschaften Leopoldina und die Union der deutschen Akademien der Wissenschaften betrachten es als ihre Aufgabe, diesen für die wissenschaftlichen Tierversuche und den Forschungsstandort Deutschland überaus wichtigen Rechtsetzungsprozess im Geiste konstruktiver Kritik zu begleiten.
Sie haben daher eine ausführliche Stellungnahme erarbeitet.

Diese finden Sie unter folgendem Link: www.leopoldina.org/fileadmin/user upload/ Politik/Empfehlungen/Nationale Empfehlungen/Leo_Stellungnahme_Tiervers_2012_ Druck.pdf

\section{Kontakt}

Deutsche Akademie der Naturforscher Leopoldina-Nationale Akademie der Wissenschaften

Postfach 110543

06019 Halle (Saale)

Tel.: $\quad+4934547239800$

E-Mail:presse@leopoldina.org 